\title{
Psicodermatologia:
}

\section{uma Interface entre Psicologia e Dermatologia}

\author{
Psychodermatology: an interface between Psychology and dermatology
}

\begin{abstract}
Resumo: O objetivo deste artigo é apresentar alguns resultados importantes provenientes da tese de doutorado da primeira autora, orientado pela segunda. O tema da tese foi um estudo com pacientes de vitiligo, através da abordagem analítica, buscando compreender o vitiligo como possibilidade de manifestação simbólica da relação psique/corpo. Treze mulheres fizeram parte do estudo, sendo que dez receberam acompanhamento médico e psicológico por seis meses. Destas, cinco receberam acompanhamento psicológico grupal e cinco, acompanhamento psicológico individual. Três pacientes receberam só acompanhamento médico por igual período. Buscou-se, com esse atendimento diferenciado, avaliar se haveria diferença à resposta clínica (condição de repigmentação) das pacientes. Os resultados indicam que o modelo analítico permitiu a compreensão simbólica da doença vitiligo como manifestação de conteúdos inconscientes que necessitavam ser integrados à consciência. As pacientes que receberam tratamento conjunto médico e psicológico apresentaram um percentual de repigmentação de até $80 \%$, enquanto as que receberam exclusivamente tratamento médico obtiveram um percentual de repigmentação de até $20 \%$. Esse dado demonstra a importância de um trabalho integrado no tratamento das doenças de manifestação psicossomática.
\end{abstract}

\section{Marisa Campio} Müller

Psicóloga. Doutora em Psicologia Clínica pela PUC-SP. Professora do

Programa de PósGraduação da PUC-RS.

\section{Denise \\ Gimenez Ramos}

Psicóloga, Doutora em Psicologia Clínica PUC-

$S P$, professora titular-

PUC-SP, coordenadora

do Curso de Pós-

Graduação em

Psicologia Clínica-PUC-

$S P$, autora dos livros A Psique do Coração e A

Psique do Corpo

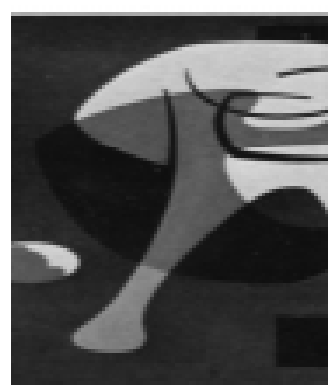

Palavras-Chave: Psicodermatologia, vitiligo, integração psique-corpo, visualização.

Abstract: The aim of this article is to present some results of the author's doctorate thesis. The theme of the thesis was a study with patients suffering from vitiligo, through the analytic approach, in order to see vitiligo as a possibility of symbolic manifestation of the psyche/body relation. Thirteen women were part of the study, whereas ten were given medical and psychological assistance for six months. Among these women, five were given group psychological therapy, and five received individual psychological therapy. Three patients were given only medical assistance for the same period. This differentiated assistance aimed at assessing if there would be any difference to the patients' clinical answer (condition of repigmentation). The results indicate that the analytical model allowed the symbolic comprehension of the vitiligo disease as a manifestation of unaware contents which needed to be integrated to consciousness. Those patients who received both medical and psychological treatment presented a higher percentage of up to $80 \%$ of repigmentation while the ones who were under medical assistance presented only $20 \%$ of repigmentation. This results showed the importance of a joint work in the psychosomatic manifestation diseases.

Key Words: Psychodermatology, vitiligo, body-mind integration, visualization.

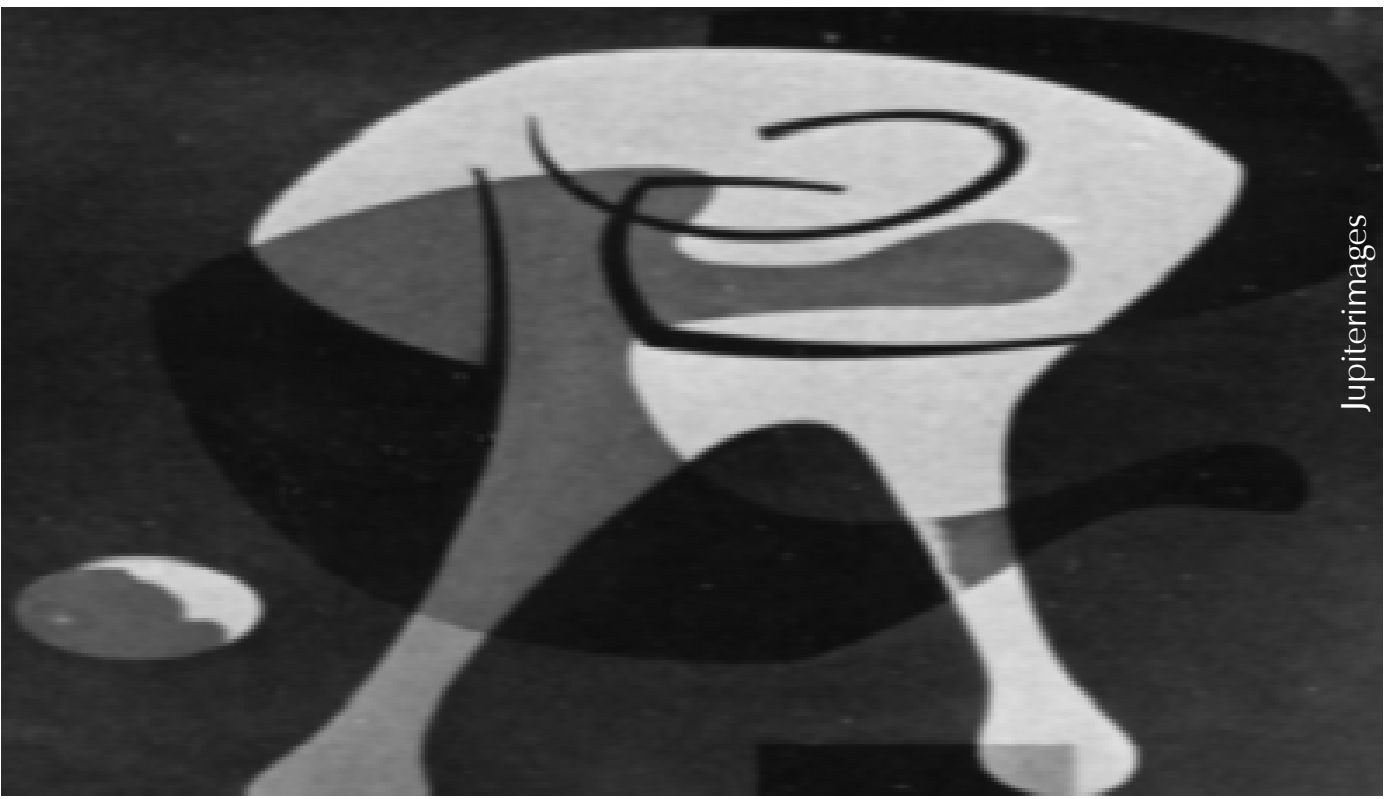




\section{Psicodermatologia: uma Visão Geral}

Como já observado através da literatura especializada, a doença vitiligo, apesar de ser uma enfermidade muito antiga, tem origem ainda desconhecida no mundo científico. No entanto, há estudos (Silvan, 2004; Müller, 2001) que apontam o surgimento do vitiligo após uma situação de estresse emocional. A literatura, no entanto, no que diz respeito aos aspectos emocionais envolvidos no vitiligo, é ainda muito limitada, necessitando de maior número de trabalhos científicos.

A Psicodermatologia é uma área que vem integrando o trabalho de médicos e psicólogos na busca por um melhor entendimento das doenças de pele (Müller et al, 2002; Koo \& Lebwhol, 2001). Os autores concordam que seja a pele o primeiro meio de contato com o mundo externo, além de um importante meio de manifestação de conflitos e emoções.

Segundo Montagu (1988), a pele é tecida com uma variedade de células resistentes e robustas e protege os tecidos macios e moles do interior do corpo. De acordo com o autor, assim como as fronteiras de uma civilização, a pele é um bastião, local em que se travam escaramuças e em que invasores encontram resistência; aí se localiza nossa primeira e última linha de defesa (p.25). São muitas as funções da pele: base dos receptores sensoriais, localização do mais delicado de todos os sentidos, o tato; fonte organizadora e processadora de informações; mediadora de sensações; barreira entre organismo e ambiente externo; fonte imunológica de hormônios para a diferenciação de células protetoras; camada protetora das partes situadas abaixo dela contra efeitos da radiação e lesões mecânicas; barreiras contra materiais tóxicos e organismos estranhos; responsável por um papel de destaque na regulação da pressão e do fluxo do sangue; órgão reparador e regenerativo; produtora de queratina; órgão de absorção de substâncias nocivas e outras, que possam ser excretadas junto com os resíduos corporais eliminados; reguladora da temperatura; órgão implicado no metabolismo de água e sal pela transpiração; reservatório de alimento e água; órgão da respiração e facilitadora da entrada e saída de gases através da mesma; sintetizador de vários compostos importantes, como a vitamina D, responsável pelo controle do raquitismo; barreira ácida que protege contra muitas bactérias; a secreção produzida pelas glândulas sebáceas lubrifica a pele e os pêlos, isolando o corpo contra chuva e frio e, provavelmente, ajuda no extermínio de bactérias; autopurificadora.
A pele pode, também, segundo Holubar (1989), ter função ego-lógica: auto-imagem, autoconfiança (excitação, medo, embaraço) e função eco-lógica: mecânica, fisioquímica (osmose), térmica, imunológica. Entre as duas funções, para o autor, e relacionadas a ambas, há as funções sensoriais, que permitem a compreensão do "self" e a experiência do "outro". Em razão dessa combinação inusitada e também da posição de interface do indivíduo com o ambiente, a pele funciona como substrato (campo de batalha) para o exógeno e o endógeno, para os processos material (físico) e psicológico.

Um suporte para manifestações simbólicas (escarificação, pintura ritual em selvagens), culturais (tatuagem e adereços metálicos), expressão dos sentimentos e troca de signos que compõem o campo de funcionamento psíquico, mediado pela linguagem (Azulay \& Azulay, 1997), é uma outra leitura possível para o entendimento da pele.

A pele também exerce o papel simbólico de proteção, o que é um fator a mais a ressaltar a vinculação entre distúrbios emocionais e as doenças da pele. Tal vínculo não data de hoje. Antigamente, já se utilizava a expressão neurodermite para demonstrar alguns tipos de eczema, com referência à sua origem nervosa.

Além de demonstrar o estado exterior e interior de nossos órgãos, a pele mostra também nossos processos e reações psíquicas em geral. Por exemplo: o rubor seria encontrado, habitualmente, em pessoas que, temendo mostrar seus sentimentos, os exprimem de forma involuntária, ficando enrubescidas. Associa-se, com freqüência, a emoções ou pensamentos proibidos, com conteúdo sexual ou agressivo. A sudorese profunda exprime um estado de ansiedade crônica (Silva, 1994).

Todavia, embora seja invisível, a condutividade elétrica da pele pode ser medida com o uso de equipamento apropriado. As experiências e as avaliações ligadas a essa área remontam a Carl Gustav Jung (1991). Dethefsen \& Dahlke (1997) investigaram os reflexos galvânicos da pele em conexão com suas experiências psicológicas utilizando a técnica de associação. Graças à eletrônica moderna, atualmente, é possível exibir as contínuas e sutis alterações da condutividade elétrica da pele e ampliá-la, a ponto de se interrogar a pessoa unicamente por meio da pele, pois cada palavra, cada pergunta, estimula a atividade elétrica da pele. Dessa forma, pode-se pensar a pele como uma grande superfície de projeção.

A pele atua, ainda, como limite dentro-fora, eu e o outro, eu e o mundo, agindo como um sistema de 
abrigo de nossa individualidade: "ao mesmo tempo que nos protege, é a fachada que nos expõe" (Strauss, 1989, p. 1221).

Anzieu (1989) segue a mesma linha de raciocínio ao afirmar que a pele é o envelope do corpo, assim como este envelopa o psíquico. É com as experiências do próprio corpo e com a mãe que a criança desenvolve seu eu psíquico, daí esse autor chamar Eu-pele essa representação que se mostra físicopsíquica.

A partir dessa idéia, surge a necessidade de investigar o vitiligo como uma possível expressão simbólica de aspectos emocionais. Essa visão bidirecional corpo/ psiquismo dá origem a um ramo da Dermatologia, a Psicodermatologia, que busca entender e tratar o paciente como um ser integral.

Apesar de vários outros trabalhos ressaltarem a manifestação de conflitos inconscientes pela pele, só mais recentemente, a partir da década de 80 , ressurge o interesse, por parte dos dermatologistas, para o estudo da relação psique/corpo. Segundo Koblenzer (1988) e Folks \& Kinney (1992), isso se deve, principalmente, à clara associação de estressores psicossociais, à baixa auto-estima e ao estigma social em pacientes com problemas dermatológicos.

Ovitiligo é, pois, uma doença sem uma etiologia definida, com prognóstico reservado e que acarreta uma série de transtornos emocionais nos pacientes. comportamental, psicoterapia de apoio, psicoterapia
No que se refere ao tratamento das doenças cutâneas, no entendimento da Psicodermatologia, além do tratamento médico, aliam-se, com freqüência, segundo Hendrickx (et al, 1991), Moffaert (1992), Folks \& Kinney (1992), Ginsburg (1996), Goleman \& Gurin (1997), Azulay \& Azulay (1997): a) tratamento psicoterápico - nas diferentes correntes: técnica de grupo; b) técnicas de relaxamento, hipnose, grupos de auto-ajuda; c) trabalho em conjunto do médico com o psicoterapeuta; d) uso de drogas psicotrópicas, principalmente ansiolíticos e antidepressivos. Essas intervenções exigem um tratamento individual - único - aquele paciente.

Como afirma Weinman (et al,1996), cada paciente tem uma representação de sua doença que é única. Esses autores sustentam que o paciente tem suas próprias crenças sobre a identidade, causa, duração e até mesmo cura da sua doença. Leventhal, citado por esses autores, propõe que essas representações refletem a resposta cognitiva do paciente para os sintomas e a doença, e que as respostas emocionais são processadas paralelamente à representação da doença.

\section{Alguns Aspectos Importantes Sobre o Vitiligo}

A incidência de vitiligo na população mundial, segundo Fitzpatrick (1997), Cao (1991), Azulay \&
Azulay (1997), Nordlund (et al, 1993), é de 1\%, afetando todas as raças e ambos os sexos. Segundo os mesmos pesquisadores, embora essa doença possa ter início em qualquer idade, em 50\% dos casos, ela tem início entre 10 e 30 anos de idade. A doença é assintomática e não afeta a sobrevivência, apesar de poder causar severo desfiguramento, levando ao estresse psicológico (Bystryn, 1997). Estudos recentes apontam dados sobre fatores de vida estressantes como desencadeadores do vitiligo (Picardi et al, 2003), além de estratégias de enfrentamento. Atualmente, já aparecem na literatura trabalhos de validação de instrumentos específicos para o vitiligo, que enfocam o desenho (Rumpf, 2004). Ainda assim, a grande maioria da literatura científica sobre o tema prima por questões exclusivamente médicas.

Como cerca de $30 \%$ dos pacientes com vitiligo têm história familiar, o fator hereditário, segundo Castenet \& Ortonne (1997), constitui uma hipótese bastante razoável. No entanto, ressalta que não se pode descartar que os membros da família também estejam expostos aos mesmos fatores ambientais, de modo que a hereditariedade do vitiligo sugere uma transmissão autossômica dominante, pois foram feitas várias análises da distribuição desse padrão, envolvendo parentesco, com a inclusão de membros secundários e terciários. Os autores ainda ressaltam que há um possível envolvimento de três a quatro alelos em tal herança poligênica. Muitos pacientes atribuem o início do vitiligo a trauma físico, doença, estresse emocional por perda de pessoas próximas ou acidentes. Um ferimento ou a reação a severas queimaduras solares pode também desencadear a doença (Fitzpatrick, 1999; Demis, 1997). Para Cao (1991), $77 \%$ dos seus pacientes desenvolveram vitiligo após estresse emocional.

O vitiligo é, pois, uma doença sem uma etiologia definida, com prognóstico reservado e que acarreta uma série de transtornos emocionais nos pacientes. O próprio surgimento do vitiligo está associado a situações de estresse emocional. Contudo, ao tratar pacientes com vitiligo, é necessário que o médico seja capaz de reconhecer que o doente não é somente a sua pele, mas um todo, e como destacam Azulay \& Azulay (1997), é um ser humano que adoece, comprometido pelo seu psiquismo.

\section{Características do estudo}

\section{Método}

A partir da literatura revisada, trata-se de um estudo de intervenção, com avaliação tipo antes e depois, que teve como objetivo principal verificar se a intervenção psicoterápica associada ao tratamento médico melhorou o estado clínico do paciente, 
levando à repigmentação (fator de efetividade). Para isso, foram selecionados 3 grupos: 1) cinco pacientes em psicoterapia individual e tratamento médico, tendo como objetivo o autoconhecimento e a ampliação da compreensão dos motivos subjacentes ao aparecimento da doença; 2) cinco pacientes em psicoterapia grupal e tratamento médico que, além dos objetivos da psicoterapia individual citados acima, tinham o grupo como meio de troca e apoio; 3) três pacientes submetidos apenas ao tratamento médico. A principal técnica utilizada durante as intervenções individuais e grupais foi a técnica de visualização (Shealy \& Myss, 1997) orientada para a condição de repigmentação. Durante as intervenções, o foco foram as questões emocionais envolvidas no surgimento da doença. As divisões entre psicoterapia individual e grupal tinham o intuito de pontuar ou não diferenças entre as modalidades de psicoterapia.

Os grupos foram acompanhados durante 6 meses.

\section{Instrumentos}

Foram utilizados, neste estudo, uma entrevista clínica que teve como objetivo colher dados biográficos que configurassem a história de vida do paciente; um questionário de qualidade de vida em pacientes com vitiligo a fim de detectar melhora da qualidade de vida após o uso da intervenção psicoterápica; avaliação médica antes e depois da intervenção psicoterápica sobre o fator repigmentação; a técnica de visualização e uma entrevista final de avaliação. Essa entrevista de avaliação foi realizada ao final dos 6 meses de tratamento, com o objetivo de avaliar individualmente qual o significado do trabalho psicoterápico frente à doença.

\section{Psicologia Analítica}

O referencial teórico que sustentou o processo psicoterápico foi o da Psicologia Analítica de C.G.Jung. Para esse autor, os sintomas são manifestações simbólicas de complexos patogênicos. A teoria junguiana prevê também a abordagem finalista na doença. Esta seria "uma reação do organismo, uma compensação, com a finalidade de levar o indivíduo a integrar o reprimido, religando o ego ao seu eixo com o Self " (Ramos, 1995, p.59).

O conceito de Self é bastante complexo no enfoque analítico, mas Jacoby (1971) refere-se a ele como o arquétipo da totalidade e o centro regulador da psique. O ego seria, então, o centro do campo da consciência e o Self a totalidade, incluindo toda a psique, os aspectos inconscientes e conscientes.

O conceito principal dessa abordagem, neste contexto, é que qualquer processo psicológico tem uma representatividade corpórea. Em casos onde os conteúdos inconscientes profundos e conflitivos não encontram representação no nível verbal, a tendência do organismo é expressá-los no nível somático, primeiramente de modo funcional e, mais tarde, anatômico (Ramos, 1995).

O vitiligo, neste trabalho, foi observado como uma representatividade na polaridade orgânica de uma disfunção do organismo como um todo, de modo que a aplicação da psicoterapia poderia levar à melhora dessa patologia por meio da conscientização dos conflitos envolvidos nesse processo.

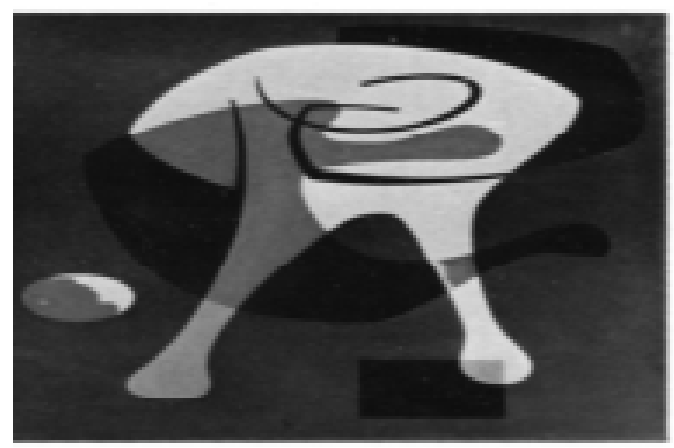

\author{
. "as imagens \\ mentais são um tipo \\ de pensamento \\ usado para fazermos \\ contato com nossa \\ realidade subjetiva \\ interna, são a \\ expressão mental de \\ um sentimento que \\ também se expressa \\ no físico.
}

Epstein

As pacientes foram atendidas num período de seis meses, caracterizando uma psicoterapia breve. Após cada sessão psicoterápica, era realizado o relaxamento e a visualização orientada para a repigmentação.

Para Epstein (1990), as imagens mentais são um tipo de pensamento usado para fazermos contato com nossa realidade subjetiva interna, são a expressão mental de um sentimento que também se expressa no físico.

Dessa forma, essa técnica foi usada buscando reforçar a relação psique/corpo.

\section{Apresentação dos resultados}

A evolução das pacientes foi avaliada quanto: 1) a conscientização dos aspectos emocionais envolvidos no vitiligo; 2) a qualidade de vida apresentada antes e depois do processo psicoterapêutico; 3 ) a condição de repigmentação alcançada.

Dentre as pacientes avaliadas, aquelas que tiveram tratamento médico e psicoterápico apresentaram até $80 \%$ de repigmentação, sendo um estudo cego, pois o médico avaliador não tinha a informação de qual paciente estava ou não em tratamento 
psicoterápico. O grupo que realizou apenas o tratamento médico apresentou, como maior índice de repigmentação, $20 \%$. Não houve diferença entre as modalidades de tratamento psicoterápico individual e grupal. Além disso, houve um aumento significativo na qualidade de vida das pacientes que realizaram o tratamento integrado.

Mesmo sem um estudo mais detalhado sobre as questões fisiológicas envolvidas no desenvolvimento e melhora da doença, resultados significativos como este demonstram claramente a inter-relação entre psique e corpo e o quanto uma intervenção em nível psicológico pode interferir na melhora física.

Outros aspectos importantes que podem ser levantados em termos de resultados são os seguintes:

- situação de estresse emocional vivida antes do aparecimento do vitiligo, como situações de perda, morte ou separação.

dificuldades de relacionamento com a figura materna: essas dificuldades estiveram basicamente relacionadas à ausência de uma figura materna nutridora e afetiva. Na quase totalidade dessas pacientes, as mães mostraram-se dominadoras, autoritárias, não afetivas e, em algumas situações, incomodadas com o papel de mãe.

figura paterna enfraquecida: foi possível identificar uma figura masculina (pai) frágil que se deixava conduzir totalmente pela mulher (mãe). É possível que a figura paterna frágil não tenha possibilitado um corte com o mundo materno, mantendo as pacientes presas à indiferenciação, privando-as da aquisição da consciência, já que esta é uma tarefa do logos paterno.

doença vitiligo como um mecanismo de defesa: tentativa da psique, como sistema auto-regulador, de estabelecer um equilíbrio entre conscienteinconsciente, mundo externo-mundo interno.
Pudemos constatar a existência de conteúdos reprimidos, somatizados em sintomas físicos, manchas de vitiligo. Podemos interpretar a despigmentação como tudo aquilo que não foi vivido, não integrado à consciência, vazios da consciência que clamam por significado.

falta de comprometimento com o tratamento médico: essa falta de comprometimento apareceu de forma importante no grupo das pacientes que não realizaram o tratamento integrado, mas esteve presente também no outro grupo, o que evidencia a necessidade de o paciente de vitiligo ter alguém o acolha e o conduza.

Cada um desses aspectos poderia ser largamente discutido aqui. Neste momento, primamos pela apresentação de resultados tão relevantes para pesquisa e para intervenção, no que tange à Psicodermatologia, em termos gerais.

\section{Considerações Finais}

Ao tratar pacientes com vitiligo, foi possível detectar alguns aspectos importantes a serem considerados na relação médico-paciente, e que caberiam ser mais detalhadamente avaliados em estudos posteriores. Trata-se de uma clientela que evita, primeiramente, o contato, mas que, ao mesmo tempo, carece de acolhimento. Demonstram descuido por si, pelo próprio corpo, pela sua saúde. As pacientes parecem ter, nesse aspecto, uma consciência muito inicial, semelhante à do bebê, que necessita ser cuidado, amparado, uma vez que ele próprio não tem condições de assumirse.

Aparece o entendimento de que, embora não seja letal, o vitiligo é uma enfermidade muito sofrida. É uma doença que trai, desfigura, expõe aos outros a dor da alma. Vem daí a necessidade de que os profissionais de saúde possam compreender a doença em um novo contexto, o da possibilidade de uma ressignificação de vida, de um ser humano mais integrado.

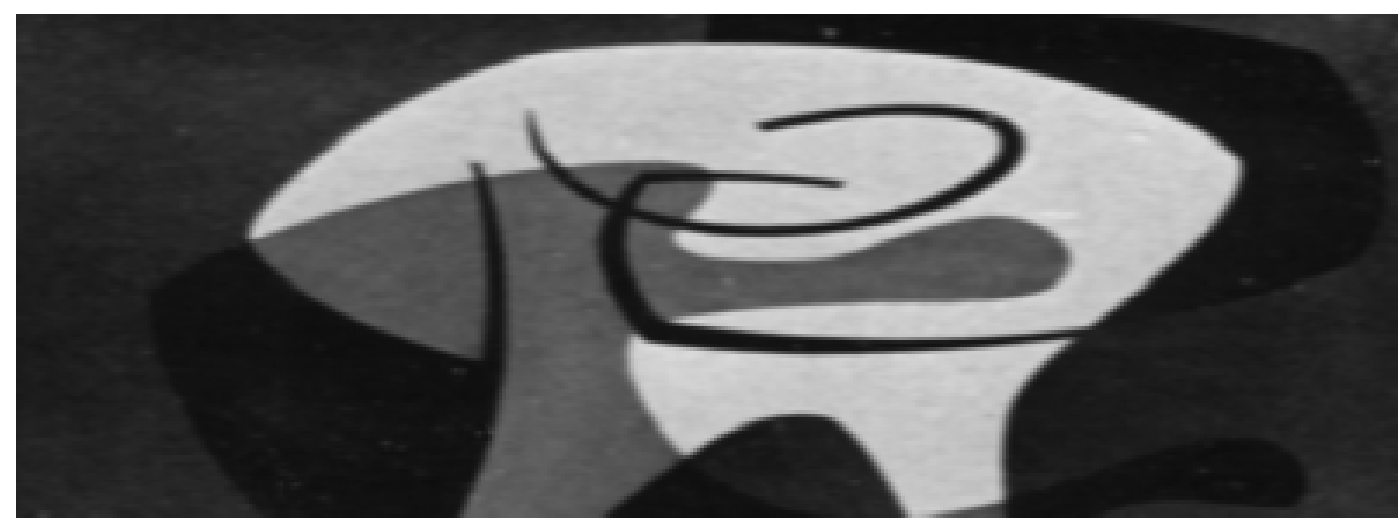


ANZIEU, D. O Eu - pele. São Paulo: Casa do Psicólogo, 1989

AZULAY, R. \& AZULAY, D. Dermatologia. Guanabara: Koogan, 1997.

BYSTRYN, J. C. Immune Mechanisms in Vitiligo. Clinicsin Dermatology, 5, n. 6, 1997,pp. 853-861.

CAO, C. M. En las Fronteras del Incurable: Histoterapia Placentaria. Avances Médicos de Cuba, Ano 1, n. 1, 1991.

CASTANET J.; ORTONNE, J.C. Pathophysiology of Vitiligo. Clinics in Dermatology, 15, n. 6, 1997, pp. 845-851.

DETHLEFSEN, T. \& DAHLKE, R. ADoença Como Caminho. São Paulo: Cultrix, 1997.

DEMIS, J. Clinical Dermatology. Philadelfia: Lippincott. Raven Publishers, 1997.

FITZPATRICK, E. Dermatology in General Medicine. USA: Mc-grawHill, 1999.

FITZPATRICK, T. \& col. Dermatology. 3. ed. New York: International Edition, 1997.

FOLKS, D. \& KINNEY, C. The Role of Psychological Factors in Dermatologic Conditions. Psychosomatic, 33, n.1, 1992, pp.42-54.

GINSBURG, I. The Psychosocial Impact of Skin Disease: an Overview. Dermatologic Clinics, 14, n. 3, 1996, pp.473 - 484.

GOLEMAN, D. \& GURIN, J. Equilíbrio Mente-corpo: Como Usar sua Mente Para uma Saúde Melhor. 3. ed. Rio de Janeiro: Campus. 1997.

HENDRICX, B. ; MOFFAERT, M. V.; SPIERS, R. \& VON FRENCKELL, Jr. The Treatment of Psychocutaneous Disorders: a New Approach. Current Therapeutic Research, 49, n.1, 1991.

HOLUBAR, K. Immunodeficiency and Skin. Why Skin? Current Problems of Dermatology, 18, 1989, pp. 298-301.

JACOBY, J. O Conceito de Ego e os Problemas de Fragilidade do Ego. Conferência proferida no Instituto Jung, Zurique, Suíça, 1971.

JUNG, C.G. A Natureza da Psique. 3oe. Petrópolis: Vozes, 1991.
KOO, J., \& LEBWHOL, A. Psychodermatology: the Mind and Skin Connection. American Family Pshysician, 64, 2001, pp. 1873-1878.

Referências

MOFFAERT, M. Psychodermatology: an Overview. Psychoterapy Psychosomatic, 58, 1992, pp.125-136.

MONTAGU, A.. Tocar o Significado Humano da Pele. São Paulo: Summus, 1988.

MULLER, M.C. Um Estudo Psicossomático de Pacientes com Vitiligo numa Abordagem Analítica. Tese de Doutorado, PUCRS, Porto Alegre, 2001.

MÜLLER, M.C., CENCl, C., HOFFMAN, F., LUDWIG, M. Os Diferentes Significados Psicológicos da Pele. Revista de Filosofia e Ciências Humanas, 18, n.1, 2002, pp. 63-73.

NORDLUNG; HARDERS, R.; GRIMES, P. Management of Vitiligo. Dermatologic Clinics, 11, n. 1, 1993, pp. 27-33.

PICARDI, A; PASQUINI P.;CATTARUZZA, M. S., GAETANO, P. Stressful Life Events, Social Support, Attachment Security and Alexithymia in Vitiligo. A Case-control Study. Psychotherapy and Psychosomatics, 72, n.3, 2003, pp.150-158.

RAMOS, D. A Psique do Corpo. São Paulo: Summus, 1995.

RUMPF H. J.; LONTZW.; UESSELER, S. A Self-administered Version of a Brief Measure of Suffering: First Aspects of Validity. Psychotherapy and Psychosomatics, 73, n.1, 2004, pp.53-56.

SHEALY, N. \& MYSS, C. Medicina Intuitiva. São Paulo: Cultrix, 1997.

SILVA, M. Quem Ama não Adoece. São Paulo: Círculo do Livro, 1994

SILVAN, M. The Psychological Aspects of Vitiligo. Cutis, 73. n. 3, 2004, pp. 163-167.

STRAUSS, G. Skin Disorders. Baltimore: Williams Wilkins, 1989.

WEINMAN, J.; PETRIE, K. \& MORRIS, R. The Illness Perception Questionnaire: a New Method for Assessing the Cognitive Representation of Illness. Psychology and Health, 11, 1996, pp. 431465. 